\title{
Study on Mechanical Design Optimization Based on Improved Particle Swarm Optimization Algorithm
}

\author{
Zhou Ning * and Zhang Jing
}

Agricultural University of Hebei; Baoding 071001, Hebei, China

\begin{abstract}
In view of local optimization in particle swarm optimization algorithm (PSO algorithm), chaos theory was introduced to PSO algorithm in this paper. Plenty of populations were generated by using the ergodicity of chaotic motion. The uniformly distributed initial particles of the particle swarms were extracted from the populations according to the Euclidean distance between particles, so that the particles could uniformly distribute in the solution space. Local search was carried out on the optimal position of the particles during evolution, so as to improve the development capability of PSO algorithm and prevent its prematurity, thus enhancing its global optimizing capability. Then the improved PSO algorithm was applied to mechanical design optimization. With optimization design for two-stage gear reducer as the study object, objective function and constraint conditions were determined by building a mathematical model of optimization design, thus realizing optimization design. Simulation and comparison between the improved algorithm and unimproved algorithm show that improved PSO algorithm can optimize the optimization results of PSO algorithm at a faster convergence rate.
\end{abstract}

Keywords: Chaos theory, mathematical model, optimization design, particle swarm optimization algorithm (pso algorithm), reducer.

\section{INTRODUCTION}

PSO algorithm which is visual, easily understood and practicable is featured by simple optimizing strategies, few debugging parameters and fast convergence rate. Therefore, it is widely applied to optimization design problems. However, this algorithm also has some limitations. It can quickly close up to the optimal value during early optimization stage, but it converges slowly near the optimal value. Its defect is local optimum. In this paper, based on the advantages of chaos theory, it is introduced to the implementation algorithm of PSO algorithm. Moreover, it is applied to mechanical design optimization. Reducer is widely applied to heavy machineries such as mining machinery, construction machinery and transportation machinery, etc. Therefore, mechanical optimization design on reducer is of great theoretical value and practical value.

\section{PARTICLE SWARM OPTIMIZATION ALGORITHM}

\subsection{Principles of Algorithm}

PSO algorithm was originally proposed by kennedy et al. [1-3]. It is mainly used for simulating bird flock's flying and foraging. The optimal result is realized by means of cooperation and competition among populations. In PSO algorithm, each candidate solution is referred to as a "particle". Firstly, the initial population is generated, and each particle is deemed as a feasible solution. The fitness is determined by the objective function. Each particle moves in the solution space, with its moving orientation and distance determined by a speed.

Generally, the particles follow the currently optimized particle, and the optimal solution will be finally obtained by searching generation by generation. The particles will trace two extremes values during each iteration process. One is the currently found optimal solution pbest; the other is the currently found optimal solution for the entire population gbest.

\subsection{Mathematical Model}

For optimization problem $[4,5]$ :

$(P) \min \left\{f(x): x\right.$ OEWÕ $\left.R^{n}\right\}, f:$ WÕ $R^{n} Æ R^{l}$

Then the set of multiple feasible solutions to $\operatorname{problem}(p)$ is referred to as a population. A feasible solution to the population is referred to as a particle, and the number of particles is referred to as population size.

The position of the $i^{\text {th }}$ particle is represented by $\mathrm{n}$ dimensional vector $X_{i}=\left(x_{i 1}, x_{i 2}, \ldots, x_{i n}\right)^{T} \mathrm{CEW}$, and the speed of the $i$ th particle is represented by $V_{i}=\left(v_{i 1}, v_{i 2}, \ldots, v_{i n}\right)^{T}$ CEW. The optimal position that a particle goes through is $P_{p i}=\left(p_{p i 1}, p_{p i 2}, \ldots, p_{p i n}\right)^{T}$ during its flying in the search space. The optimal positions that all particles go through are indicated by index number $g$, i.e., $P_{g}$. Therefore, the speed 
of the particles as well as the positions of the evaluation function of the calculation function in each generation can be transformed according to Formula (2) and Formula (3) [6]:

$v_{i d}(t+1)=v_{i d}(t)+c_{1} \operatorname{rand}_{1} *\left(p_{\text {pid }}-x_{i d}(t)\right)+$

$c_{2} \operatorname{rand}_{2} *\left(p_{g d}-x_{i d}(t)\right)$

$x_{i d}(t+1)=x_{i d}(t)+v_{i d}(t+1)$

where: $i=1,2, \ldots, m ; d=1,2, \ldots, n ;$ rand $_{1}$ and rand $_{2}$ are random numbers which are distributed subject to $U(0,1)$; learning factors $c_{1}$ and $c_{2}$ are nonnegative constants, $c_{1}=c_{2}=2 ; v_{i d}=\left[-v_{\max }, v_{\max }\right], v_{\text {max }}$ is the upper limit of the speed defined by user.

The iteration process of the algorithm is as follow [7]:

Initialize population: random $X_{i}$

repeat:

for each particle $i \mathrm{OE}[1, S]$

if $f\left(X_{i}\right)<f\left(P_{i}\right)$

$P_{i}=X_{i}$

end

if $f\left(P_{i}\right)<f\left(P_{g}\right)$

$P_{g}=P_{i}$

end

update the position and velocity of particle using equation (2) and equation (3)

end

until termination criterion is satisfied

\section{IMPROVED PSO ALGORITHM}

In view of local optimization in PSO algorithm, chaos theory was introduced to PSO algorithm in this paper to improve the algorithm. The algorithm flow is as follow [810]:

Step1: chaos initialization. Assume that the variable to be optimized is a $D$-dimensional one. A $D$-dimensional vector will be randomly generated $z_{1}=\left[z_{11}, z_{12}, \ldots, z_{1 D}\right]$, each component is in the scope $[0,1]$. Then $M$ components are generated according to Logistic equation [8], $z_{1}, z_{2}, \ldots, z_{M}$ :

$z_{n+1}=m z_{n}\left(1-z_{n}\right), n=0,1,2, \ldots ; 0<z_{n}<1 ; m \mathrm{EE}[0,4]$
The chaos interval is mapped to the value range of the variables according to Formula (5)

$x_{i j}=a_{j}+\left(b_{j}-a_{j}\right) z_{i j}$

where $b_{j}, a_{j}$ represent the upper and lower limits of the optimized variables respectively.

Step2: calculate the fitness values of each particle according to the objective function. Select $N$ particle swarms with good performance from $M$ initial particle swarms, the speeds of the particles are generated randomly.

Step3: set the initial individual extreme values and the global extreme values for the particles. Define the current position of each particle as individual extreme values $P_{i}$, calculate the fitness values corresponding to $P_{i}$ (the individual extreme values) according to the objective function, select the positions of the particles with the best values to define the global extreme values $P_{g}$.

Step 4: update the flying speed and positions of the particles according to the updating formulas of speed and position.

Step 5: carry out chaos optimization for the optimal position $P_{g}$. Firstly, map the optimal positions to the definitional domain [0,1] of Logistic equation [11], i.e., using Formula (6). Then iterate according to Logistic equation to generate $m$ chaos variable sequences. Finally, map the chaos variable sequences generated to the value interval of the optimized variables to generate $m$ particles. Calculate the fitness values of each particle to acquire optimal solution $p$ '.

$z_{9}=\frac{P_{g}-a_{i}}{b_{i}-a_{i}}$

Step 6: replace $p^{\prime}$ with the position of any particle in the current population.

Step 7: return to Step4, stop calculation until the ending condition for the particle swarm is met, put out the calculation results.

\section{MODEL FOR REDUCER OPTIMIZATION DESIGN}

Take a two-stage gear reducer as the study object, its organization diagram is as shown in Fig. (1) [12].

This design optimization problem consists of 7 design variables, width of the gear surface, $x_{1}$; gear die, $x_{2}$; number of pinions, $x_{3}$; bearing spacing of axle $1, x_{4}$; bearing spacing of axle $2, x_{5}$; diameter of axle $1, x_{6}$; diameter of axle $2, x_{7}$. The value range of the design variables is: 


$$
\begin{aligned}
& 2.6 £ x_{1} £ 3.6,0.7 £ x_{2} £ 0.8,17 £ x_{3} £ 28, \\
& 7.3 £ x_{4} £ 8.3,7.3 £ x_{5} £ 8.3,2.9 £ x_{6} £ 3.9, \\
& 5.0 £ x_{7} £ 5.5
\end{aligned}
$$

The objective of the objective function is the minimum volume of the reducer:

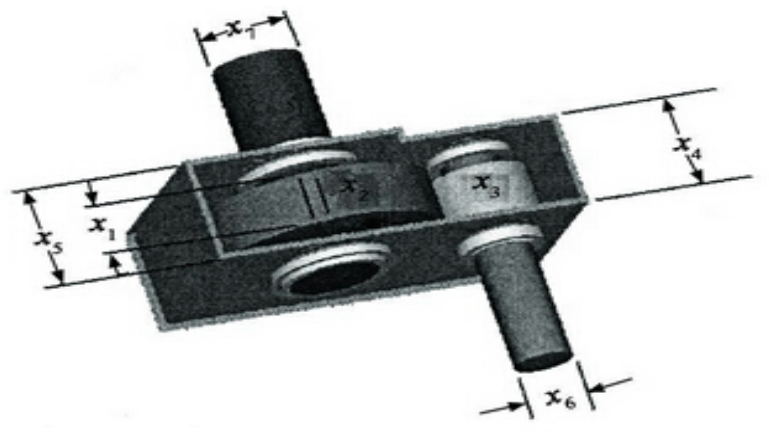

$g_{9}(x)=1.9-x_{5}+1.5 x_{7} £ 0$

$g_{10}(x)=A_{1} B_{1}^{-1}-1800 £ 0$

$g_{11}(x)=A_{2} B_{2}^{-1}-1800 £ 0$

\section{SIMULATION EXPERIMENT}

To verify the effectiveness of the algorithm in this paper, the aforesaid mathematical model is optimized and solved by using the methods proposed in this paper. The maximum number of iterations is 50 , the population size is 20 , popmin $=-5.12, \quad \operatorname{popmax}=5.12, \quad \operatorname{vmax}=1, \quad \operatorname{vmin}=-1$, the simulation result is as shown in Fig. (2):

The optimization results of the improved PSO algorithm are as follows: design variable $(3.5,0.7,17,7.30$, $7.7153,3.3502,5.2867)$, its convergence graph is as shown in Fig. (2).

Fig. (1). Organization diagram of the reducer.

$$
\begin{aligned}
& \min f 1(x)=0.7854 x_{1} x_{2}^{2}\left(3.3333 x_{3}^{2}+14.933 x_{3}-43.0934\right)-1.508 x_{1}\left(x_{6}^{2}+x_{7}^{2}\right)+7.477\left(x_{6}^{3}+x_{7}^{3}\right)+ \\
& 0.7854\left(x_{4} x_{6}^{2}+x_{5} x_{7}^{2}\right)
\end{aligned}
$$$$
A_{1}=\left[\left(745 x_{2}^{-1} x_{3}^{-1} x_{4}\right)^{2}+16.9 ¥ 10^{6}\right]^{0.5}
$$$$
B_{1}=0.1 x_{6}^{3}
$$$$
A_{2}=\left[\left(745 x_{2}^{-1} x_{3}^{-1} x_{5}\right)^{2}+157.5 ¥ 10^{6}\right]^{0.5}
$$$$
B_{2}=0.1 x_{7}^{3}
$$

Constraint conditions include 11 constraint conditions of bending stress and contact stress of the gear, lateral deviation of the axle as well as design dimension, etc. $[13,14]$ :

$$
\begin{aligned}
& g_{1}(x)=27 x_{1}^{-1} x_{2}^{-2} x_{3}^{-1}-1 £ 0 \\
& g_{2}(x)=397.5 x_{1}^{-1} x_{2}^{-2} x_{3}^{-2}-1 £ 0 \\
& g_{3}(x)=1.93 x_{2}^{-1} x_{3}^{-1} x_{4}^{3} x_{6}^{-4}-1 £ 0 \\
& g_{4}(x)=1.93 x_{2}^{-1} x_{3}^{-1} x_{5}^{3} x_{7}^{-4}-1 £ 0 \\
& g_{5}(x)=x_{2} x_{3}-40 £ 0 \\
& g_{6}(x)=5-x_{1} x_{2}^{-1} £ 0 \\
& g_{7}(x)=x_{1} x_{2}^{-1}-12 £ 0 \\
& g_{8}(x)=1.9-x_{4}+1.5 x_{6} £ 0
\end{aligned}
$$

To compare the differences between improved PSO algorithm and PSO algorithm, the parameters are set as follows: maximum number of iterations is 100 , population size is 20 , $\operatorname{popmin}=-5.12$, $\operatorname{popmax}=5.12, \operatorname{vmax}=1$, vmin $=-1$. The comparison diagram of the convergences before and after improvement is as shown in Fig. (3):

According to Fig. (3), the convergence rate of the improved PSO algorithm is remarkably faster and more stable than that of PSO algorithm, which can verify the stability and effectiveness of the algorithm in this paper.

\section{CONCLUSION}

The study object in this paper is design optimization of reducer. In view of local optimization in PSO algorithm, chaos theory is introduced to PSO algorithm. A chaos PSO algorithm is proposed to carry out the optimization design. The algorithm flow of chaos PSO algorithm is expounded in details, then optimization and simulation design are carried out based on specific cases of optimization design for gear reducer. The results show that the algorithm in this paper has certain theoretical value and practical value as its convergence rate is superior to that of PSO algorithm; therefore, it can be promoted for engineering application.

\section{CONFLICT OF INTEREST}

The authors confirm that this article content has no conflict of interest.

\section{ACKNOWLEDGEMENTS}

Declared none. 
(a) First Computational Results

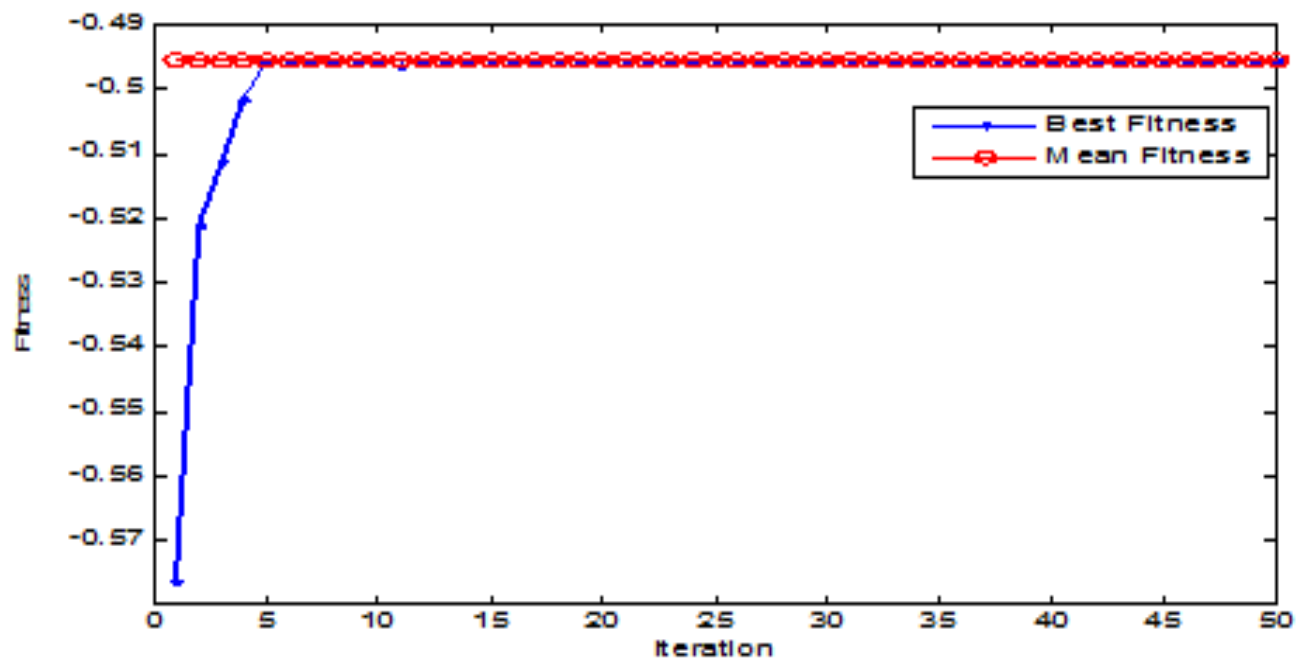

(b) Second Computational Results

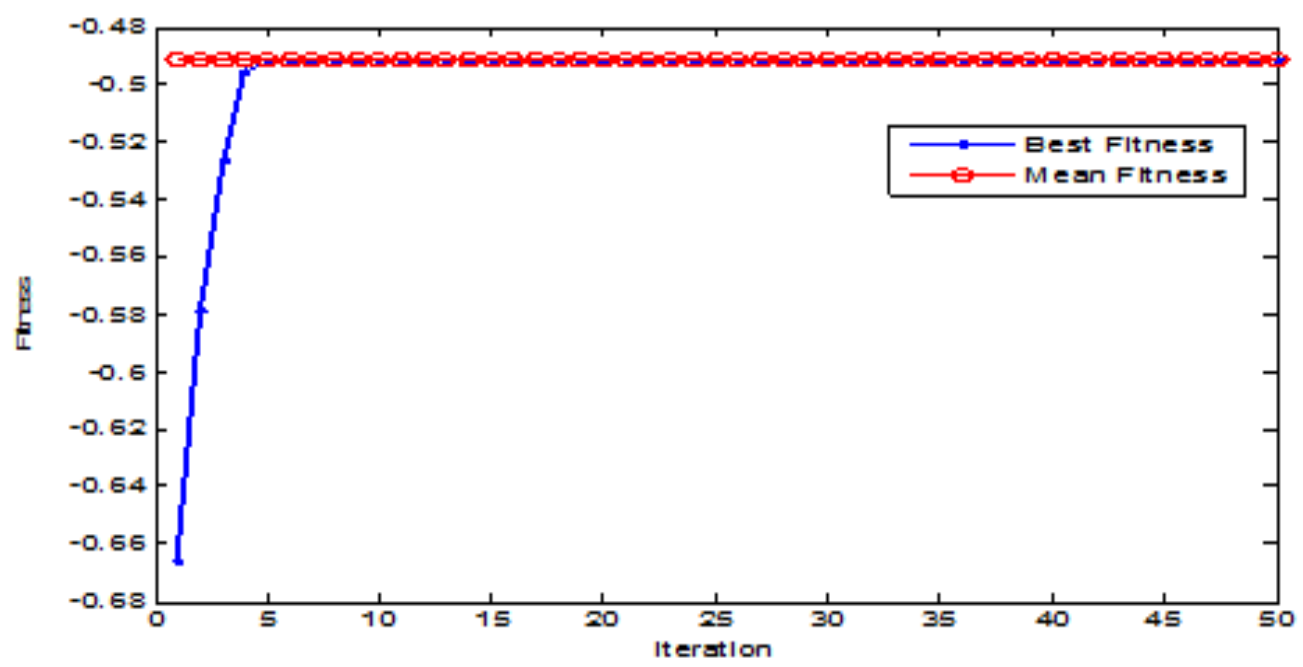

(c) Third Computational Results

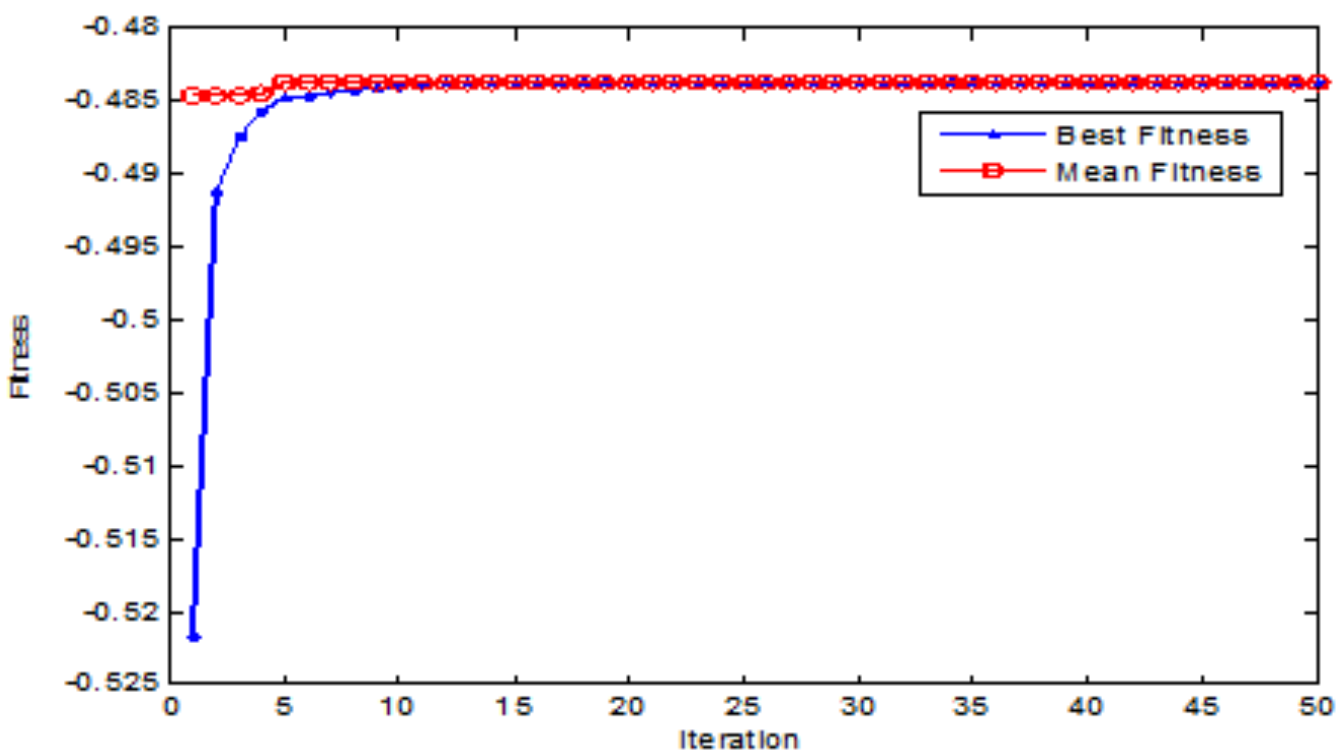

Fig. (2). The solving results of the improved PSO. 


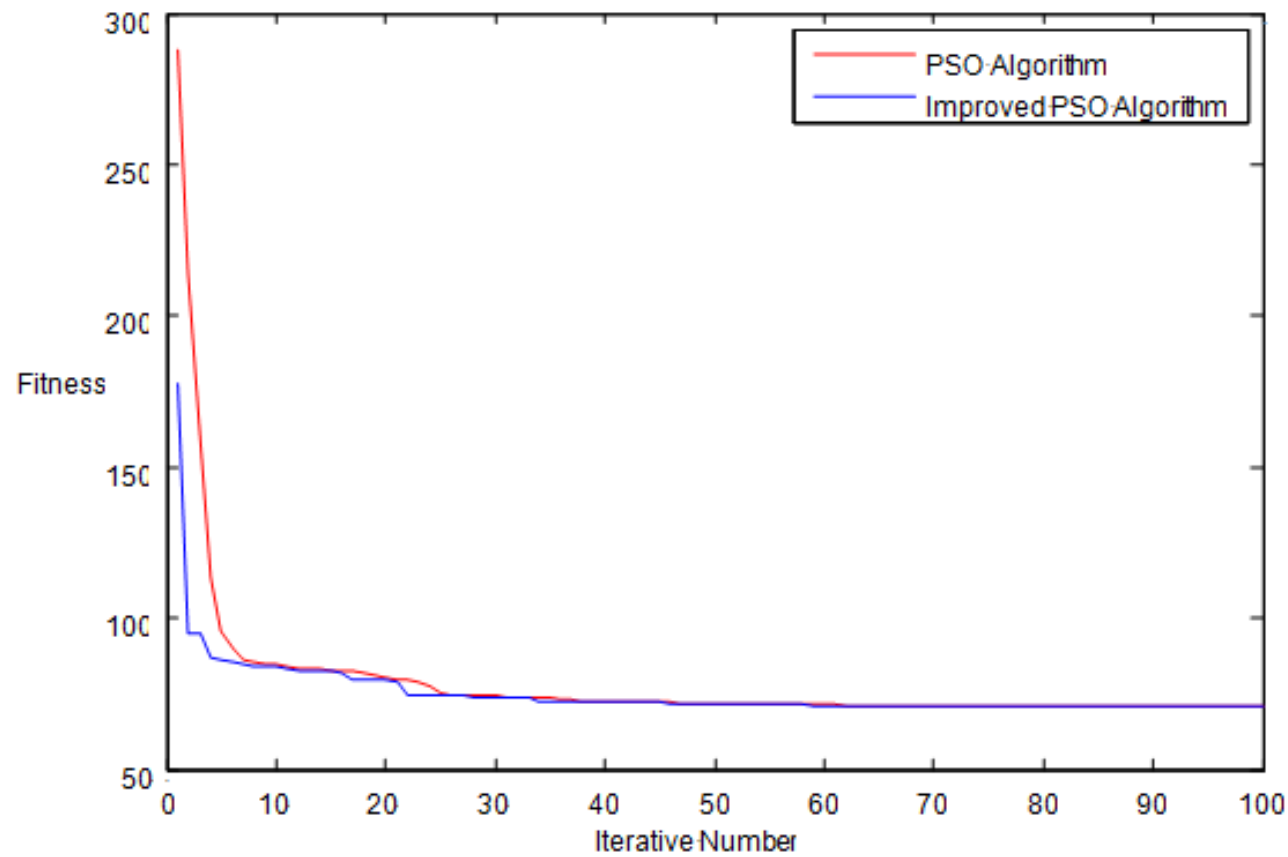

Fig. (3). The comparison diagram of the convergences of the improved PSO algorithm and PSO algorithm.

\section{REFERENCES}

[1] D. L. Donoho, "Compressed sensing," IEEE Transactions on Information Theory, vol.52, no.4, pp.1289-1306, 2006.

[2] E. Candes, and J. Romberg, "Quantitive robust uncertainty principles and optimally sparse decompositions," Foundations of Compute Math, vol. 6, no.2, pp.227-254, 2006.

[3] J.N. Laska, S. Kirolos, M.F. Duarte, T.S. Ragheb, R.G. Baraniuk, Y. Massoud, "Theory and implementation of an analog to information converter using random demodulation", In: Proceedings of the IEEE International Symposium on Circuits and Systems (ISCAS), Piscataway: Institute of Electrical and Electronics Engineers Inc, pp. 1959-1962, 2007.

[4] T. Joel, and G. Anna, "Signal recovery from random measurements via orthogonal matching pursuit," IEEE Transactions on Information Theory, vol. 53, no.12, pp. 4655-4666, 2007.

[5] D. Donoho, and Y. Tsaig, "Fast Solution of Ell-1-Norm Minimization Problems when the Solution may be Sparse," Technical Report, Stanford University Department of Statistics, 2006.

[6] A. T. F. Mário, D. N. Robert, and J. W. Stephen, "Gradient projection for sparse reconstruction: Application to compressed sensing and other inverse problems," IEEE Journal of Selected Topics in Signal Processing: Special Issue on Convex Optimization Methods for Signal Processing, vol.1, no. 4, pp. 586-598, 2007.

[7] K. Egiazarian, A. Foi, and V. Katkovnik, "Compressed sensing image reconstruction via recursive spatially adaptive filtering", In: IEEE International Conference on Image Processing (ICIP), San Antonio, TX, 2007, pp. I-549-I-552. 2007.
[8] M. Duarte, M. Davenport, D. Takhar, J. Laska, T. Sun, K. Kelly, and R. Baraniuk, "Single-pixel imaging via compressive sampling,” IEEE Signal Processing Magazine, vol. 25, no.2, pp. 83-91, 2008.

[9] M. Wakin, J. Laska, M. Duarte, D. Baron, S. Sarvotham, D. Takhar, K. Kelly, and R. Baraniuk, "An architecture for compressive imaging," International Conference on Image Processing (ICIP), Atlanta, Georgia, 2006.

[10] M. Wakin, J. Laska, M. Duarte, Dror. Baron, S. Sarvotham, D. Takhar, K. Kelly, and R. Baraniuk, "Compressive imaging for video representation and coding", In: Proceedings on Picture Coding Symposium (PCS), Beijing, China, 2006.

[11] D. Takhar, J. Laska, M. Wakin, M. Duarte, D. Baron, S Sarvotham, K. Kelly, and R. Baraniuk, "A new compressive imaging camera architecture using optical-domain compression", Computational Imaging IV at SPIE Electronic Imaging, San Jose, California, 2006.

[12] J. Haupt and R. Nowak, "Compressive sampling vs conventional imaging", International Conference on Image Processing (ICIP), Atlanta, Georgia, 2006.

[13] R. Maleh, A. Gilbert, and M. Strauss, "Sparse gradient image reconstruction done faster," In: IEEE Conference on Image Processing (ICIP), San Antonio, Texas, 2007.

[14] K. Egiazarian, A. Foi, and V. Katkovnik, "Compressed sensing image reconstruction via recursive spatially adaptive filtering," In: IEEE Conference on Image Processing (ICIP), San Antonio, Texas, 2007.

(C) Ning and Jing; Licensee Bentham Open.

This is an open access article licensed under the terms of the (https://creativecommons.org/licenses/by/4.0/legalcode ), which permits unrestricted, non-commercial use, distribution and reproduction in any medium, provided the work is properly cited. 